\title{
The patient journey map: Improving the emergency department communication experience for patients and their family and friends.
}

\section{Stephanie VandenBerg, Heather Hair, Gillian Harvey, Eddy Lang, David Stringer}

Background: The 2013 Urban and Regional Emergency Department (ED) Patient Experience Report indicates that the most important factor influencing a patients' ED experience is a combination of staff care and communication. Lack of communication in the emergency room experience can be addressed by design methods and processes. The Emergency Strategic Care Network $^{\mathrm{TM}}$ assembled an interdisciplinary team of experts from various clinical, academic, and information design backgrounds to engage patients, families and providers to improve the ED intake experience. This innovative partnership resulted in the development of a graphic information system that directs, informs and educates patients in EDs in Alberta.

Implementation: Using the Plan-Do-Study-Act (PDSA) framework, focus groups were conducted to understand the communication needs of emergency department patients. An information design specialist co-created a graphic information system (the patient journey map) and worked with AHS communications to ensure it met AHS guidelines. Patients were then approached to participate in a 14-question survey about the usability and accuracy of the journey map as well as the impact it had on their ED visit.

Our team consisted of Heather Hair, Executive Director of the (ESCN) who provided leadership in identifying communication as a key area for improvement and coordinating ED partners and patient involvement. Gillian Harvey is an assistant professor of Design Studies at the University of Alberta. She used the data collected in focus groups to design a 2-D communication map. David Stringer acted as project manager for the implementation and evaluation of the journey map. Stephanie Vandenberg is an emergency physician and was responsible for designing the evaluation strategy including research methods and data analysis. An official journey map is now available to print for emergency departments across Alberta.

Evaluation Methods: The objective was to understand what information ED patients require during their visit to better understand the process by which they are triaged and receive care. Data collection consisted of a 10-minute, 14 question interview. Each question allowed for positive, neutral or negative feedback to capture unintended consequences of the journey map. Quantitative demographic and journey map-specific variables were collected and reported as frequencies. Qualitative data was analyzed using thematic analysis with thematic codes developed and assigned to the qualitative responses. Both quantitative and qualitative analysis was undertaken by two members of the research team. Responses were analyzed against the demographic variable of age category to determine if age impacts communication needs and desired medium of communication in the ED.

Results: Seven hospitals took part in this survey, conducted between September 1, 2019 and May 5, 2020. 162 emergency department patients participated. Most people agreed that the 
journey map clarified the ED patient process and accurately reflected their experience of the ED journey. The journey map did not seem to make the wait less confusing. Participants reported the journey map was good at helping them understand the overall emergency department intake process and did a good job of helping them understand the reason for waiting/delays. The journey map was excellent at helping the participant understand why specific tests/treatments were needed but was poor at helping them to understand the total time it would take them to be seen. 